\title{
Finding MFE Structures Formed by Nucleic Acid Strands in a Combinatorial Set
}

\author{
Mirela Andronescu and Anne Condon \\ The Department of Computer Science, U. British Columbia \\ andrones,condon@cs.ubc.ca
}

\section{Introduction}

When designing sets of DNA strands for biomolecular computations, it is often desirable to have a "structure free" combinatorial set of strands, that is, a set of long strands which do not form any secondary structure, obtained by concatenating short strands in the designed set.

The ability to computationally predict the combination in a combinatorial set of strands with lowest minumum free energy (MFE) secondary structure is also useful in design of strands for directed mutagenesis and SELEX experiments [3] - biochemical analyses of a library of nucleic acid sequences, to determine whether simple mutations of the sequences have desired properties. The input sequence sets can be represented as strings of characters (DNA or RNA nucleotides) and "wild cards", which can code for several different characters using IUPAC (International Union of Pure and Applied Chemistry) format or other format. In the case of SELEX, it is useful to be able to predict not only the combination whose minimum energy structure has lowest energy, but also other combinations with minimum energy structures of low value; this is the problem we address in this paper.

In earlier work, we described an algorithm, CombFold, that calculates which concatenated long strand in a combinatorial set forms the minimum free energy secondary structure with the lowest energy [2]. In this work, we extend that algorithm to output $k$ secondary structures with the lowest minimum free energies, where $k$ is specified by the user.

We use the following definitions and notation throughout.

- Let word denote an RNA or DNA sequence $w=v_{1} v_{2} \ldots v_{l}$, where $v_{i} \in\{A, C, G, U\}$ for RNA and $v_{i} \in\{A, C, G, T\}$ for DNA. The orientation of the strand is from 5' to 3', unless otherwise stated. For example, ACGCUAGGCA is an RNA word of length 10.

- Let set denote a set of $g$ words of the same length $l$. Formally we use the notation $S=\left\{w_{1}, w_{2}, \ldots, w_{g} \mid \operatorname{length}\left(w_{i}\right)=\operatorname{length}\left(w_{j}\right), \forall i, j \in\right.$ 
$\{1, \ldots, g\}, i \neq j\}$. The following set (displayed as a column of words) is formed of 4 words of length 5 :
AUACG
UAGCG
GCCGA
CUGCG

The word order in a set does not matter, but for convenience later, we assume that the words in $S$ are indexed and can be ranked by their index.

- Let Input-Set denote a sequence of $s$ sets, $I S=S_{1}, S_{2}, \ldots, S_{s}$. For example, the following is an Input-Set of 5 sets:

$\begin{array}{lllll}\text { UAGCGA } & \text { CAGCGUAAUAU } & \text { AUGCG } & \text { AUAGCGGUA } & \text { AUCG } \\ \text { AUAGAU } & \text { AGAUGCGCGGU } & & \text { GAGCGCAAG } & \text { CUGC } \\ & \text { UAGGCUAGCGU } & & & \text { GCGA }\end{array}$

Note that the number of words in each set can differ, as can the length of the words across sets.

An Input-Set can also be written in terms of words rather than sets: $I S=$ $\left\{w_{i j}, 1 \leq i \leq s, 1 \leq j \leq g_{i}, \forall i, 1 \leq i \leq s\right\}$, where $g_{i}$ is the number of words in the set $i$.

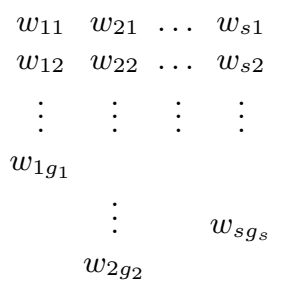

Thus, an Input-Set IS is characterized by $s$ sets, where each set $S_{i}$ has $g_{i}$ words, of length $l_{i}$. In what follows, when $I S$ is fixed, we consider all its characteristics: $s, w_{i j}, g_{i}, l_{i}, \forall i, j, 1 \leq i \leq s, 1 \leq j \leq g_{i}$, to be known.

- Let Combination denote an RNA/DNA sequence, formed by concatenating one word $w_{i j}$ of each set $S_{i}$ from $I S$, starting at $S_{1}$ and finishing at $S_{s}$. For example $C=w_{11} w_{21} \ldots w_{s 1}$ is a combination formed by concatenating the first word of each set together. Generally, a combination is of the form $C=w_{1 b_{1}} w_{2 b_{2}} \ldots w_{s b_{s}}$, where $1 \leq b_{i} \leq g_{i}$. Here, $b_{i}$ denotes the word rank within the set $S_{i}$. A combination has the length $n=\sum_{i=1}^{s} l_{i}$. If we think of a combination as a sequence of nucleotides rather than a concatenation of words, we can denote it as $C=c_{1} c_{2} \ldots c_{n}$, with $c_{i} \in\{A, C, G, U\}$ for RNA.

- Given an Input-Set IS, the set of all possible combinations forms the Combinatorial-Set: $C S=\left\{w_{1 b_{1}} w_{2 b_{2}} \ldots w_{s b_{s}} \mid 1 \leq b_{i} \leq g_{i}\right\}$. Note that 
all combinations have the same length: $n=\sum_{i=1}^{s} l_{i}$ and that $C S$ has $g_{1} \times g_{2} \times \ldots \times g_{s}$ elements. If $g_{i}>1, \forall i$, then the number of elements in $C S$ is exponential in $s$.

The optimal MFE combination problem is: given an RNA Input-Set IS and a thermodynamic model $M$, predict which combination, out of all elements of the Combinatorial-Set CS formed from $I S$, folds to a pseudoknot-free secondary structure with the lowest minimum free energy.

An extension of the optimal MFE combination problem is to find the $k$ best MFE combinations, rather than the optimal one only. The $k$-suboptimal MFE combinations problem is: given an RNA Input-Set IS and a thermodynamic model $M$, predict which $k$ different combinations, out of all elements of the Combinatorial-Set CS formed from $I S$, fold to pseudoknot-free secondary structures with the lowest minimum free energies.

In this paper, we build on earlier work [2] to develop an algorithm for the $k$-suboptimal MFE combination problem. In section 2, we first review our dynamic programming algorithm which runs in polynomial time, for solving the optimal MFE combination problem. Then, in Section 3, we present our algorithm for the $k$-suboptimal MFE combinations problem. We provide a theoretical and empirical analysis of the optimal and $k$-suboptimal MFE combinations problems in Section 4 and show that both run in polynomial time.

\section{Review of Algorithm for the Optimal MFE Combination Problem}

Our CombFold algorithm [2] is based on the classical algorithm of Zuker and Stiegler [4] for finding the minimum free energy secondary structure of a single RNA strand.

One method to solve the optimal MFE combination problem is to create all possible combinations and then to run the Zuker-Stiegler algorithm on each of them. However, depending on the characteristics of the Input-Set, the number of combinations may be very big. If $g_{i}=g>1, \forall i$, then there are $g^{s}$ combinations. Since the Zuker-Stiegler algorithm runs in $\Theta\left(n^{3}\right)$ time, where $n$ is the length of the combinations, this approach has running time complexity that is $\Theta\left(g^{s} n^{3}\right)$. More generally, the number of combinations is exponential in the number of sets which have at least two words. We have implemented this exhaustive search approach under the name of ExhaustS, which will be discussed in Section 4.

To avoid this exponential running time, we extended the Zuker-Stiegler algorithm. In the description that follows, we use indices $i$ and $j$ for the nucleotide positions (i.e. columns in Table 1 ) in a combination $C$. We use $s(i)$ and $s(j)$ to denote the sets in which $c_{i}$ and $c_{j}$ are positioned, respectively. We say that $c_{i}$ and $c_{j}$ belong to, or are in, the sets $s(i)$ and $s(j)$ respectively. 


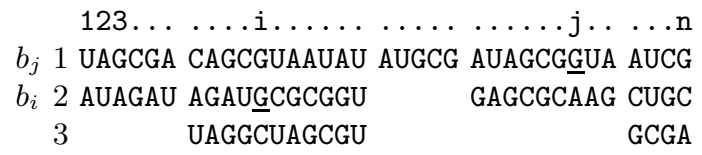

Table 1. Example of a combinatorial set of short RNA sequences.

We use $b_{i}$ and $b_{j}$ to denote the indices (i.e. the rows in Table 1 ) of the words containing $c_{i}$ and $c_{j}$ within the sets $s(i)$ and $s(j)$. Given a set $S, g(S)$ returns the number of words in $S$. Hence, $b_{i}$ can take $g(s(i))$ values. When the InputSet $I S$ and $b_{i}$ are given, we let the base $c_{i}$ at position $i$ of a combination that is in column $i$ and row $b_{i}$ be given by the function $c_{i}=\operatorname{Nucleotide}\left(I S, b_{i}, i\right)$. Table 1 shows an example of the nucleotides $c_{i}$ and $c_{j}$.

\section{Notation for substructure free energy values}

We use the following notation to denote free energy values of various substructures; in our implementation, the values are stored in four-dimensional arrays.

- $W^{\prime}(j)$ is the lowest minimum free energy of a structure formed from the first $j$ nucleotides $c_{1} c_{2} \ldots c_{j}$ of a combination. Consequently, $W^{\prime}(n)$ contains the lowest minimum free energy of any structure formed by any combination in the Combinatorial-Set corresponding to the Input-Set IS.

- $W^{c}\left(b_{j}, j\right)$ is the lowest minimum free energy of a structure formed from the first $j$ nucleotides of a combination in which $b_{j}$ is the word index of the set $s(j)$.

- $V^{c}\left(b_{i}, b_{j}, i, j\right)$ is the lowest minimum free energy of a structure formed from a combination fragment $c_{i} \ldots c_{j}$ starting at $i$ and ending at $j$, and with fixed word indices $b_{i}$ and $b_{j}$, assuming that $\left(c_{i} . c_{j}\right)$ is a base pair.

- $H^{c}\left(b_{i}, b_{j}, i, j\right)$ is the lowest free energy of a combination fragment $c_{i} \ldots c_{j}$ in which $b_{i}$ and $b_{j}$ are fixed, assuming that $\left(c_{i} \cdot c_{j}\right)$ closes a hairpin loop.

- $S^{c}\left(b_{i}, b_{j}, i, j\right)$ is the lowest free energy of a combination fragment $c_{i} \ldots c_{j}$ in which $b_{i}$ and $b_{j}$ are fixed, assuming that $\left(c_{i} . c_{j}\right)$ closes a stacked loop.

- $V B I^{c}\left(b_{i}, b_{j}, i, j\right)$ is the lowest minimum free energy of the combination $c_{i} \ldots c_{j}$ in which $b_{i}$ and $b_{j}$ are fixed, assuming that $\left(c_{i} . c_{j}\right)$ closes an internal loop.

- $V M^{c}\left(b_{i}, b_{j}, i, j\right)$ is the lowest minimum free energy of a combination fragment $c_{i} \ldots c_{j}$ in which $b_{i}$ and $b_{j}$ are fixed, assuming that $\left(c_{i} . c_{j}\right)$ closes a multi-branched loop.

- $W M^{c}\left(b_{i}, b_{j}, i, j\right)$ is the lowest minimum free energy value of a combination fragment $c_{i} \ldots c_{j}$ that forms part of a multi-branched loop, and is used to calculate $V M^{c}$ values. 


\section{Recurrence relations}

The array free energy values are calculated using several recurrence relations. In describing these here, we omit for clarity the calculations involving dangling ends and terminal AU penalties [1]. First, $W^{\prime}(j)$ is the minimum of the values $W^{c}\left(b_{j}, j\right)$, over all possible $b_{j}$ :

$$
W^{\prime}(j)=\min _{b_{j}} W^{c}\left(b_{j}, j\right) .
$$

Here,

$$
W^{c}\left(b_{j}, j\right)=\min \left\{\begin{array}{c}
\min _{b_{j-1} \in X\left(\left\{b_{j}, j\right\},\{j-1\}\right)} W^{c}\left(b_{j-1}, j-1\right) \\
\min _{1 \leq i<j ; b_{i-1}, b_{i} \in X\left(\left\{b_{j}, j\right\},\{i-1, i\}\right)} \\
\left(V^{c}\left(b_{i}, b_{j}, i, j\right)+W^{c}\left(b_{i-1}, i-1\right)\right)
\end{array}\right.
$$

where $X$ is a function which returns the feasible range of words for all of the needed (unknown) indexes. For the first line, the word corresponding to $j-1$ depends on the sets to which $j$ and $j-1$ belong, and on $b_{j}$ :

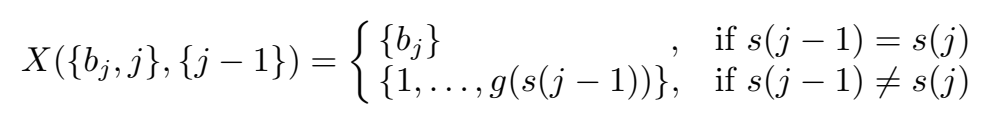

For the second line of the recurrence for $W^{c}\left(b_{j}, j\right)$, there are two word indices, $b_{i-1}$ and $b_{i}$, that we have to find the ranges for:

$$
\begin{aligned}
& X\left(\left\{b_{j}, j\right\},\{i-1, i\}\right)= \\
& \begin{cases}b_{j}, b_{j} & , \text { if } s(i-1)=s(i)=s(j) \\
\{1, \ldots, g(s(i-1))\}, b_{j} & , \text { if } s(i-1) \neq s(i)=s(j) \\
\{1, \ldots, g(s(i-1))\}, b_{i-1}, & \text { if } s(i-1)=s(i) \neq s(j) \\
\{1, \ldots, g(s(i-1))\},\{1, \ldots, g(s(i))\}, & \text { if } s(i-1) \neq s(i) \neq s(j)\end{cases}
\end{aligned}
$$

In the first two lines of the equation for $W^{c}$ above, the feasible values for $b_{j-1}$ (first line), and $b_{i-1}, b_{i}$ (second line), depend on one other index: $j$, and its corresponding $b_{j}$. However, in a more general case, there are $p$ indexes with known $b$ 's, and $q$ indexes with unknown $b$ 's, for which we want to find the feasible ranges. The number of if lines needed to specify the function $X$ in the general case will be $2^{p+q-1}$. Since we are using the nearest neighbour thermodynamic model, the highest values for $p$ and $q$ are $p=4$ and $q=4$ in the case of internal loops, and $p=2$ and $q=6$ in the case of multi-branched loops, yielding $2^{7}=128$ if lines. Instead of enumerating all of these lines in our code, we developed an algorithm to compute the ranges for unknown b's, for arbitrary values of $p$ and $q$. This procedure is described next.

The function $X$ calculates the ranges for the unknown $b$ 's, for any number of known and unknown indexes. Figure 1 gives the pseudocode for the $X$ procedure. The input is comprised of two groups: the first group contains 


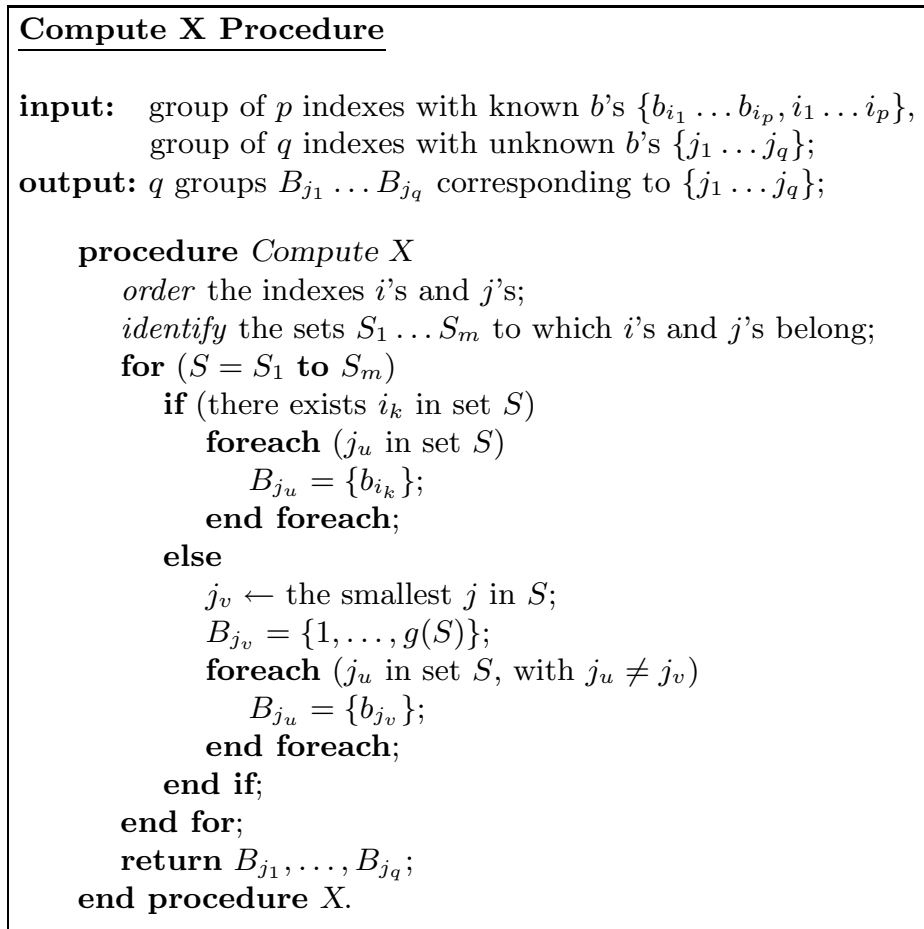

Procedure 1: Pseudocode for the X procedure. Details are described in the text.

the known $b$ 's and the known indexes: $\left\{b_{i_{1}} \ldots b_{i_{p}}, i_{1} \ldots i_{p}\right\}$. The input has the property that if $s\left(i_{j}\right)=s\left(i_{j+1}\right)$ then $b_{i_{j}}=b_{i_{j+1}}$. The known $b$ 's help to determine the ranges of the unknown $b$ 's. The second group contains the indexes of the unknown $b$ 's, $\left\{j_{1} \ldots j_{q}\right\}$. First, we need to order all the values $i_{1} \ldots i_{p}, j_{1} \ldots j_{q}$. This is necessary for the second step, which identifies the sets corresponding to each index. The two extreme situations are: (1) all indexes are in the same set, and thus there will be only one possible configuration for the unknown $b$ 's; (2) all indexes are in different sets, hence there will be $g\left(s\left(j_{1}\right)\right) \times \ldots \times g\left(s\left(j_{q}\right)\right)$ possible values for the unknown $b$ 's.

Once we have identified the sets, for each set $S$, first we check whether there exists the index of a known $b$ in this set. If so, then all the $j$ 's in $S$ will have the corresponding, unknown, $b$ 's equal to the known $b$. No other option is available for these unknown $b$ 's, since the value for the known $b$ is fixed. If no known $b$ exists in $S$, then all the unknown $b$ 's in $S$ will be in the range $\{1, \ldots, g(S)\}$, with the constraint that they will have the same values, being in the same set. In other words, we can give a value to the $b$ of the smallest index in $S$, and all the other b's in $S$ will have the same value. The function $X$ will return a group of values for the needed unknown $b$ 's. 
An example of a particular situation, with the groups $\left\{b_{i}, b_{j}, i, j\right\}$ and $\{i+1, i+2, j-2, j-1\}$ as input, is presented in Table 2 , where $s(i) \neq$ $s(i+1)=s(i+2) \neq s(j-2) \neq s(j-1)=s(j)$. In this case, $b_{i+1}$ will take values in the range $\{1, \ldots, g(s(i+1))\}, b_{i+2}$ will take the value that $b_{i+1}$ takes, $b_{j-2}$ will be in the range $\{1, \ldots, g(s(j-2))\}$, and $b_{j-1}$ equals $b_{j}$. Hence, for this particular situation, there will be $g(s(i+1)) \times g(s(j-2))$ terms over which to minimize.

\begin{tabular}{c|cc|c|cc}
$i$ & $i+1$ & $i+2$ & $j-2$ & $j-1$ & $j$ \\
\hline $\mathbf{b}_{\mathbf{i}}$ & 1 & $b_{i+1}$ & 1 & $b_{j}$ & $\mathbf{b}_{\mathbf{j}}$ \\
& $\vdots$ & & $\vdots$ & & \\
& $g(s(i+1))$ & & $g(s(j-2))$ & &
\end{tabular}

Table 2. Example of choices for $b$ values for a particular situation. The known $b$ 's are in bold. The vertical lines signify that the index to the left is in a different set from the index to the right.

Using function $X$ to decide which are the possible values for each word, the remaining recurrence relations for CombFold are a logical extension of the corresponding recurrence relations for the Zuker-Stiegler algorithm. The recurrences use free energy values for hairpins, stacked pairs, and interior loops which we denote by $\Delta G-H^{c}\left(I S, b_{i}, b_{j}, i, j\right), \Delta G-S^{c}\left(I S, b_{i}, b_{j}, b_{i+1}, b_{j-1}, i, j\right)$, and $\Delta G-I^{c}\left(I S, b_{i}, b_{j}, b_{i^{\prime}}, b_{j^{\prime}}, i, j, i^{\prime}, j^{\prime}\right)$, respectively.

The relations for $V^{c}$ and $H^{c}$ are straightforward:

$$
\begin{gathered}
V^{c}\left(b_{i}, b_{j}, i, j\right)=\left\{\begin{array}{cc}
+\infty & \text { for } i \geq j \\
\min \left(H^{c}\left(b_{i}, b_{j}, i, j\right), S^{c}\left(b_{i}, b_{j}, i, j\right),\right. & \\
\left.V B I^{c}\left(b_{i}, b_{j}, i, j\right), V M^{c}\left(b_{i}, b_{j}, i, j\right)\right), & \text { for } i<j
\end{array}\right. \\
H^{c}\left(b_{i}, b_{j}, i, j\right)=\Delta G-H^{c}\left(I S, b_{i}, b_{j}, i, j\right)
\end{gathered}
$$

We omit the details of the calculation of hairpin free energies; the interested reader can find these in the M.Sc. thesis of Andronescu [1]. For the calculation of stacked loops, finding $b_{i+1}$ and $b_{j-1}$ is imposed again by the nearest neighbour model itself.

$$
\begin{gathered}
S^{c}\left(b_{i}, b_{j}, i, j\right)=\min _{b_{i+1}, b_{j-1} \in X\left(\left\{b_{i}, b_{j}, i, j\right\},\{i+1, j-1\}\right)} \\
\left(\Delta G-S^{c}\left(I S, b_{i}, b_{j}, b_{i+1}, b_{j-1}, i, j\right)+V^{c}\left(b_{i+1}, b_{j-1}, i+1, j-1\right)\right) .
\end{gathered}
$$

The internal loop free energy calculation is a minimization over $i^{\prime}$ and $j^{\prime}$, i.e. the closing pair of the internal loop. Once $i^{\prime}$ and $j^{\prime}$ fixed, we calculate the free energy value for each possible $b_{i^{\prime}}$ and $b_{j^{\prime}}$ : 


$$
\begin{gathered}
V B I^{c}\left(b_{i}, b_{j}, i, j\right)=\min _{i<i^{\prime}<j^{\prime}<j}\left(\min _{b_{i^{\prime}}, b_{j^{\prime}} \in X\left(\left\{b_{i}, b_{j}, i, j\right\},\left\{i^{\prime}, j^{\prime}\right\}\right)}\right) \\
\left.\left(\Delta G-I^{c}\left(I S, b_{i}, b_{j}, b_{i^{\prime}}, b_{j^{\prime}}, i, j, i^{\prime}, j^{\prime}\right)+V^{c}\left(b_{i^{\prime}}, b_{j^{\prime}}, i^{\prime}, j^{\prime}\right)\right)\right)
\end{gathered}
$$

The free energy for multi-loops adds the minimization over the necessary $b$ 's as well. The equations for $W M^{c}$ and $V M^{c}$ follow, where $\mathcal{M}_{a}, \mathcal{M}_{b}$, and $\mathcal{M}_{c}$ are penalties for multi-loops, branches, and unpaired bases that determine the standard multi-loop energy function. For $i<j$,

$$
\begin{aligned}
& W M^{c}\left(b_{i}, b_{j}, i, j\right)=\min \\
& \left\{\begin{array}{l}
V^{c}\left(b_{i}, b_{j}, i, j\right)+\mathcal{M}_{b}, \\
\min _{b_{i+1} \in X\left(\left\{b_{i}, b_{j}, i, j\right\},\{i+1\}\right)}\left(W M^{c}\left(b_{i+1}, b_{j}, i+1, j\right)+\mathcal{M}_{c}\right), \\
\min _{b_{j-1} \in X\left(\left\{b_{i}, b_{j}, i, j\right\},\{j-1\}\right)}\left(W M^{c}\left(b_{i}, b_{j-1}, i, j-1\right)+\mathcal{M}_{c}\right), \\
\min _{i \leq h<j ; b_{h}, b_{h+1} \in X\left(\left\{b_{i}, b_{j}, i, j\right\},\{h, h+1\}\right)}\left(W M^{c}\left(b_{i}, b_{h}, i, h\right)+\right. \\
\left.W M^{c}\left(b_{h+1}, b_{j}, h+1, j\right)\right)
\end{array}\right. \\
& V M^{c}\left(b_{i}, b_{j}, i, j\right)=\mathcal{M}_{a}+\min _{i<h<j-1 ; b_{i+1}, b_{h}, b_{h+1}, b_{j-1} \in X\left(\left\{b_{i}, b_{j}, i, j\right\},\{i+1, h, h+1, j-1\}\right)} \\
& \left(W M^{c}\left(b_{i+1}, b_{h}, i+1, h\right)+W M^{c}\left(b_{h+1}, b_{j-1}, h+1, j-1\right)\right)
\end{aligned}
$$

In the implementation of our software CombFold v1.0, we did not implement the equation for $V M^{c}$ as described above. This equation contains the sum of two $W M^{c}$ terms in order to make sure that the multi-loop obtained has at least three branches (including the closing one), at the cost of increased complexity, i.e. $n^{3}$ instead of $n^{2}$ for computing $V M^{c}$ (see also section 4). In our implementation, $V M^{c}=\mathcal{M}_{a}+W M^{c}\left(b_{i+1}, b_{j-1}, i+1, j-1\right)$ where $b_{i+1}, b_{j-1} \in X\left(\left\{b_{i}, b_{j}, i, j\right\},\{i+1, j-1\}\right)$, while we used a mechanism to make sure that the predicted multi-loops have at least three branches. We believe that this does not involve significantly different predictions, and we plan to implement the more accurate formula above in the next version of CombFold.

\section{An algorithm for the $k$-suboptimal MFE combinations problem}

The algorithm for the optimal MFE combination problem, just described in the previous section, returns only the combination which has the smallest MFE. We next describe how the algorithm can be extended to return the $k$ combinations that have the lowest MFE.

Suppose that the Input-Set IS contains $s$ sets $S_{i}$, each having $g_{i}$ words. We will add the superscript "(1)" to the notation of our sets to denote that first 
we are looking for the optimal combinations. The superscripts for the next combinations will be " $(2)$ " and so on. Thus, $I S^{(1)}=\left\{S_{1}^{(1)}, S_{2}^{(1)}, \ldots, S_{s}^{(1)}\right\}$ will be associated with the Combinatorial-Set $C S^{(1)}$.

First, we find the optimal MFE combination using the method described in the previous section. Let the combination $C^{(1)}=w_{1 C_{1}}^{(1)} w_{2 C_{2}}^{(1)} \ldots w_{s C_{s}}^{(1)}$ denote the optimal MFE combination, where $C_{i}$ denotes the index of the word in the set $S_{i}^{(1)}$, which belongs to the optimal combination. The Input-Set I $S^{(1)}$ contains all the possible combinations of the original set $I S$. To find the next best combinations, first we partition the set $I S^{(1)}$ into $s$ sets which do not contain $C^{(1)}$ :

$$
\begin{aligned}
& I S^{(2) 1}=\left\{S_{1}^{(1)}-\left\{w_{1 C_{1}}^{(1)}\right\}, \quad S_{2}^{(1)}, \ldots, \quad, S_{s}^{(1)}\right\} \\
& I S^{(2) 2}=\left\{\begin{array}{c}
(1) \\
\left.1 C_{1}\right\},
\end{array} S_{2}^{(1)}-\left\{w_{2 C_{2}}^{(1)}\right\}, \ldots, \quad S_{s}^{(1)}\right\} \\
& \vdots \\
& I S^{(2) s}=\left\{\quad\left\{w_{1 C_{1}}^{(1)}\right\}, \quad\left\{w_{2 C_{2}}^{(1)}\right\}, \ldots, S_{s}^{(1)}-\left\{w_{s C_{s}}^{(1)}\right\}\right\}
\end{aligned}
$$

For convenience later, we denote the newly created sets with $S_{i}^{(2) j}$, where $1 \leq i, j \leq s, i$ denotes the set index within the Input-Set, as in the previous notations, and $j$ denotes the index of the newly created Input-Set:

$$
\begin{aligned}
& I S^{(2) 1}=\left\{S_{1}^{(2) 1}, S_{2}^{(2) 1}, \ldots, S_{s}^{(2) 1}\right\} \\
& I S^{(2) 2}=\left\{S_{1}^{(2) 2}, S_{2}^{(2) 2}, \ldots, S_{s}^{(2) 2}\right\} \\
& \vdots \\
& I S^{(2) s}=\left\{S_{1}^{(2) s}, S_{2}^{(2) s}, \ldots, S_{s}^{(2) s}\right\}
\end{aligned}
$$

The Input-Sets $I S^{(2) 1}, I S^{(2) 2}, \ldots, I S^{(2) s}$ have the following properties:

- $C^{(1)} \notin C S^{(2) m}, \forall m, 1 \leq m \leq s$;

- $C S^{(2) m} \cap C S^{(2) m^{\prime}}=\emptyset, \forall m, m^{\prime}, 1 \leq m, m^{\prime} \leq s, m \neq m^{\prime}$;

- $\left\{C^{(1)}\right\} \cup C S^{(2) 1} \cup \ldots \cup C S^{(2) s}=C S^{(1)}$,

where $C S^{(i) j}$ denotes the Combinatorial-Set associated with the Input-Set $I S^{(i) j}$. In other words, (1) the combination $C^{(1)}$ is not included in any of the new Input-Sets created by the partitioning process, (2) the new input sets do not have any combinations in common and (3) the whole space of combinations in $C S^{(1)}$ is covered by the new input sets plus the optimal combination found. This leads to finding the optimal combinations for each of $I S^{(2) 1}, I S^{(2) 2}, \ldots, I S^{(2) s}$, followed by choosing the one with the smallest MFE. Thus, the free energy of the second best combination, i.e. the combination with the second lowest MFE, will be $\Delta G^{(2)}=\min \left(\Delta G^{(2) 1}, \Delta G^{(2) 2}, \ldots \Delta G^{(2) s}\right)$, 


$$
\begin{aligned}
& C^{(1)} \rightarrow \Delta \mathrm{G}^{(1)} \ldots, \ldots, \ldots
\end{aligned}
$$

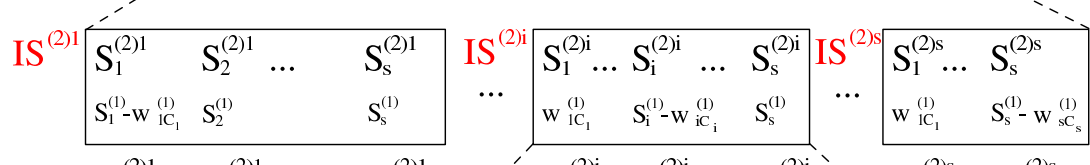

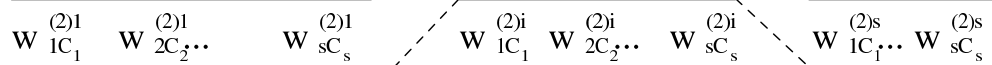

$$
\begin{aligned}
& \Delta \mathrm{G}^{(2) 1} \quad \mathrm{\prime}^{\prime} \quad \Delta \mathrm{G}^{(2) \mathrm{s}} \quad \because \Delta \mathrm{G}^{(2) \mathrm{s}} \\
& C^{(2)} \rightarrow \min \left(\Delta \mathrm{G}^{(2) 1}, \ldots, \Delta \mathrm{G}^{(2) \mathrm{i}}, \ldots, \Delta \mathrm{G}^{(2) \mathrm{s}}\right) \stackrel{\text { assume }}{=} \Delta \mathrm{G}^{(2) \mathrm{i}} \\
& \operatorname{IS}^{(3) 1} \begin{array}{lll}
\mathrm{S}_{1}^{(3) 1} & \ldots & \mathrm{S}_{\mathrm{s}}^{(3) 1} \\
\mathrm{~S}_{1}^{(2)}-\mathrm{w}_{1 \mathrm{C}_{1}}^{(2 \mathrm{i}} & & \mathrm{S}_{\mathrm{s}}^{(2)} \\
\hline \mathrm{W}_{1 \mathrm{C}_{1}}^{(3) 1} & \ldots & \mathrm{W}_{\mathrm{SC}_{\mathrm{s}}}^{(3) 1}
\end{array} \\
& \Delta G^{(3) 1} \\
& \begin{array}{c}
\ddots \\
\ddots
\end{array} \\
& \ldots \quad \begin{array}{ll}
\mathrm{IS}^{(3) \mathrm{s}} \mathrm{s} \\
\mathrm{S}_{1}^{(3) \mathrm{s}} \ldots & \mathrm{S}_{\mathrm{s}}^{(3) \mathrm{s}} \\
\mathrm{w}_{1 \mathrm{C}_{1}}^{(2 \mathrm{i}} & \mathrm{S}_{\mathrm{s}}^{(2) i}-\mathrm{w}_{\mathrm{sc}_{\mathrm{s}}}^{(2) i}
\end{array} \\
& \mathrm{~W}{ }_{{ }_{1} \mathrm{C}_{1}}^{(3) \mathrm{s}} \ldots \mathrm{W}_{\mathrm{SC}}^{(3) \mathrm{s}} \\
& \triangle G^{(3) s} \\
& C^{(3)} \rightarrow \min \left(\Delta \mathrm{G}^{(2) 1}, \ldots, \Delta \mathrm{G}^{(2) \mathrm{i}-1}, \Delta \mathrm{G}^{(2)+1+1} \ldots, \Delta \mathrm{G}^{(2) \mathrm{s}}, \Delta \mathrm{G}^{(3) 1}, \ldots, \Delta \mathrm{G}^{(3) \mathrm{s}}\right)
\end{aligned}
$$

Fig. 1. The algorithm for finding the $k$ suboptimal MFE combinations of a combinatorial set.

where $\Delta G^{(2) i}$ is the MFE of the optimal combination of $I S^{(2) i}$. Let $i$ be such that $\Delta G^{(2)}=\Delta G^{(2) i}$ and let $C^{(2)}=w_{1 C_{1}}^{(2) i} w_{2 C_{2}}^{(2) i} \ldots w_{s C_{s}}^{(2) i}$ denote the second best combination. The next step is to partition $I S^{(2) i}$, in the same way we partitioned $I S^{(1)}$. We will obtain the Input-Sets $I S^{(3) 1}, I S^{(3) 2}, \ldots I S^{(3) s}$. Now, note that the following are true:

- $C^{(1)}$ and $C^{(2)} \notin C S^{(2) m}$ and $C S^{(3) m^{\prime}}, \forall m, m^{\prime}, 1 \leq m, m^{\prime} \leq s, m \neq i$;

- $C S^{(a) m} \cap C S^{(b) m^{\prime}}=\emptyset$, for $a, b \in\{2,3\}$ and $m, m^{\prime} \in\{1, \ldots, s\}$, with either $a \neq b$ or $m \neq m^{\prime}$ (or both);

- $\left\{C^{(1)}, C^{(2)}\right\} \cup C S^{(2) 1} \cup \ldots \cup C S^{(2) i-1} \cup C S^{(2) i+1} \cup \ldots \cup C S^{(2) s} \cup C S^{(3) 1} \cup$ $\ldots \cup C S^{(3) s}=C S^{(1)}$.

Thus, $\Delta G^{(3)}$, the MFE of the third combination, will be

$$
\min \left(\Delta G^{(2) 1}, \ldots, \Delta G^{(2) i-1}, \Delta G^{(2) i+1}, \ldots, \Delta G^{(2) s}, \Delta G^{(3) 1}, \ldots, \Delta G^{(3) s}\right) .
$$

Figure 1 shows the steps just described. Recursively continuing in the same way, we can find the best $k$ combinations. However, note that the tree of partitioned Input-Sets will grow proportionally with $k$, more exactly, it will have a number of leaves that is at most $k s$, which implies increase in run time and space. 
It is important to note that when creating the new Input-Sets $I S^{(i) j}$, $1 \leq j \leq s$ the sets with a lower index $j$ will typically have a bigger solution space (i.e. number of possible combinations) than the ones with a higher index. Thus, if after we found the second combination, $\Delta G^{(2)}$ equals $\Delta G^{(2) s}$, the third combination will be found much more quickly than if $\Delta G^{(2)}$ equals $\Delta G^{(2) 1}$. Also, it is possible that the Input-Set which has the next best combinations will be partitioned in less than $s$ partitions (or even no partitions at all), since the other partitions are empty. In this case, only the optimal MFE combinations of the non-empty partitions will be considered. Examples of the running time on some problem instances are discussed in Section 4.

\section{Time and space complexity}

Extending the $O\left(n^{3}\right)$ algorithm for secondary structure prediction of single nucleic acid molecules, the optimal MFE combination algorithm traverses the Input-Set in the same way, but for each position $i$ and $j$, several possibilities might exist. We consider that the number of words $g_{i}$ in each set $S_{i}$ is limited by a constant bound $g_{\max }$, and we measure the complexity in terms of the combinations length: $n=l_{1}+l_{2}+\ldots+l_{s}$. Also, we consider that the ranges returned by the $X$ function is bounded by a constant and will be omitted from the theoretical analysis. In practice, the number of words in each set, the number of sets, the length of the words in each set, as well as the nucleotides composing the set, all have an impact on the run time. First we give an analysis of the theoretical complexity, and later in this section we will analyse the CombFold implementation on several specific Input-Sets.

\section{Theoretical analysis}

The theoretical time complexity of calculating each array described in Section 2 in the worst case follows:

- $W^{\prime}: O\left(g_{\max } n\right)$, because for each $j$ calculated in $W^{c}$, we minimize over all possible words of $j$, and there are at most $g_{\max }$ such words;

- $W^{c}: O\left(g_{\max }^{5} n^{2}\right)$, because for each $j, 1 \leq j \leq n$ there are at most $g_{\max }$ possibilities, and we minimize over $i$. When dangling ends are included, $i$ and $j$ 's neighbours may have unknown $b$ 's, leading to four options for unknown $b$ 's (details omitted). However, $b_{i-1}, b_{i}$ and $b_{i+1}$ can only be in different words if the length of the word $l(s(i))$ is 1 . But if $l(s(i))=1$, $g(s(i))$ is at most 4 (because there are 4 different nucleotides), no matter what the value of $g_{\max }$ is;

- $V^{c}: O\left(g_{\max }^{2} n^{2}\right)$, because for each $i$ and $j$, we minimize over a constant number of terms, and for each $i$ and $j$ there are at most $g_{\max }$ possibilities;

- $S^{c}: O\left(g_{\max }^{4} n^{2}\right)$, because for each $i, j$ and their corresponding $b_{i}$ and $b_{j}$, we minimize over potential different values for $b_{i+1}$ and $b_{j-1}$; 
- $H^{c}: O\left(g_{\text {max }}^{4} n^{2}\right)$, because for each $i, j$ and their corresponding $b_{i}$ and $b_{j}$, the term which has the greatest complexity has minimization over 4 terms, but 2 of them happen only if the word length is 1 , so they are reduced to constant times;

- $V B I^{c}: O\left(g_{\max }^{8} n^{4}\right)$, but we assume the internal loops do not have more than a constant number of bases (e.g. 30) on each side between the branches, and thus the complexity for internal loops becomes $O\left(g_{\max }^{8} n^{2}\right)$. The power of 8 comes from the most general case of internal loops;

- $W M^{c}: O\left(g_{\max }^{4} n^{3}\right)$, because the most costly branch of the $W M^{c}$ calculation for each $i$ and $j$ is to find the best $h$ for multi-loop partitioning. Each of $i, j$ and $h$ are in at most $g_{\max }$ words;

- $V M^{c}: O\left(g_{\max }^{8} n^{3}\right)$, because for each $i$ and $j$ we minimize over $h$, and when we include all dangling ends, there are two known $b$ 's and six unknown $b$ 's in the worst case.

Thus, if we consider both $g_{\max }$ and $n$ in our analysis, the worst case time complexity is $O\left(g_{\max }^{8} n^{3}\right)$. In practice, $g_{\max }$ is often considered a constant, which leads to complexity proportional to $n^{3}$. The arrays $W^{\prime}, W^{c}, V^{c}$ and $W M^{c}$ need to be stored in memory. The space complexity is $O\left(g_{\max }^{2} n^{2}\right)$, or $O\left(n^{2}\right)$ if we consider $g_{\max }$ a constant.

The worst theoretical time complexity of the $k$-suboptimal MFE combinations problem is $O\left(s k g_{\max }^{8} n^{3}\right)$ and the worst space complexity is $O\left(s k g_{\max }^{2} n^{2}\right)$. However, in practice, some of the Input-Sets after partitioning become empty.

\section{Empirical analysis}

We compared the running time performance of CombFold v1.0 with suboptimal predictions with that of ExhaustS, a simple (exponential time) exhaustive search algorithm, which creates all possible combinations and for each, calculates its minimum free energy using SimFold [1], our implementation of the Zuker-Stiegler algorithm. For Input-Sets with a small number of combinations, it is expected that CombFold takes more time and space than ExhaustS, because CombFold is a more complex algorithm. However, although the space is not a problem for ExhaustS, the running time quickly grows and becomes impractical.

Figure 2 gives the run time performance of CombFold with $k=1,2,3,10$ and ExhaustS on randomly generated Input-Sets of different characteristics. All the tests have been performed on machines with CPU Pentium III 733 $\mathrm{MHz}$, memory cache $256 \mathrm{~KB}$ and RAM memory $1 \mathrm{~GB}$, running Linux 2.4.20. All graphs show the CPU time in seconds, presented on a log scale, versus variation of different characteristics of the Input-Sets. To simplify the analysis, we chose $g_{1}=\ldots=g_{s}=g$ and $l_{1}=\ldots=l_{s}=l$, and we took variations of $s, g$ and $l$. Having all set sizes equal and all set lengths equal, the number of combinations will be $g^{s}$, and the length of the combinations will be $l \cdot s$.

The graph in (a) shows a comparison between the running time of CombFold with $k=1,2,3,10$ and ExhaustS, on a set of 19 instances having $g$ and 
$l$ fixed at 2 and 10, respectively. The number of sets $s$ varies from 1 to 19 , yielding $2^{1}=2$ combinations of length 10 to $2^{19} \approx 0.5 \cdot 10^{6}$ combinations of length 190. CombFold with $k=1$ becomes faster than ExhaustS at $s=8$, with $k=2$ and 3 becomes faster at $s=10$, and CombFold with $k=10$ becomes faster at $s=12$. Note that the slope of the curves suggest that CombFold grows polynomially, while Exhaust $S$ grows exponentially in $s$.

The graph in (b) shows a similar situation as in graph (a), but when $g$ is fixed at 3 rather than $2, l=10$ and $s$ takes values in the range 1 to 12 , leading to $3^{1}=3$ combinations of length 10 to $3^{12} \approx 0.5 \cdot 10^{6}$ combinations of length 120. The number of combinations being bigger for the same $s$, CombFold with $k=1$ outperforms Exhaust $S$ when $s=6$, with $k=2$ and 3 when $s=7$, and with $k=10$ when $s=8$.

Graph (c) shows a comparison when $s$ and $l$ are fixed to 6 and 10 respectively, but $g$ varies from 1 to 13 . These yield $1^{6}=1$ to $13^{6} \approx 4.8 \cdot 10^{6}$ combinations of length 60 . Note that in this case ExhaustS grows polynomially in $g$, however, it grows more quickly than CombFold. Indeed, the graph shows that CombFold with $k=1$ becomes faster than the ExhaustS when $g=3$, with $k=2$ and 3 when $g=4$ and with $k=10$ when $g=5$.

Graph (d) gives the comparison when $s$ and $g$ are fixed to 8 and 2, respectively, leading to a fixed number of $2^{8}=256$ combinations. However, the length of the words vary from 10 to 100 , yielding combinations of length 80 to 800 . Again, ExhaustS grows more quickly, but still polynomially, only the length of the combinations being changed. Exhaust $S$ is outperformed by $\operatorname{CombFold}(k=1)$ at $l=10$ and by $\operatorname{CombFold}(k=2)$ at $l=50$. On the instances we tested, ExhaustS outperforms CombFold with $k=10$, and becomes roughly the same speed as CombFold with $k=3$ when $l=100$.

On all these four graphs, we note that CombFold with $k=1$ and 2 , and ExhaustS are nicely curved, while CombFold with $k=3$ and 10 have "hills" and "valleys". To see how the curves look like, we created two sets of 50 instances of Input-Sets with exactly the same characteristics: graph (e) with $s=10, g=3, l=5$ and graph (f) with $s=8, g=8, l=4$. The results comfirm the explanation we gave earlier in Section 3: When $k=1$, CombFold fills all the arrays, a small variation happening due to the distribution of the nucleotides in the words. When $k=2$, the arrays for $s$ more sets are always calculated, no matter what the optimal combination is. However, depending on which the second best combination is, the size of the next Input-Sets that partition the solution space can differ substantially. This influence propagates on to the next best combinations, such that when $k=10$, the differences in time between different instances can vary substantially. Also, note that for some instances, the time for $k=3$, and even for $k=10$, is very close or equal to the time for $k=2$. This means that the second best combination was part of a very small Input-Set, which was partitioned in fewer (or even 0) non-empy Input-Sets. The graphs also show the run time of the exponential algorithm. For graph (e) there are $3^{10} \approx 60,000$ combinations of length 50 , and Exhaust $S$ is more than one order of magnitude slower than CombFold with 


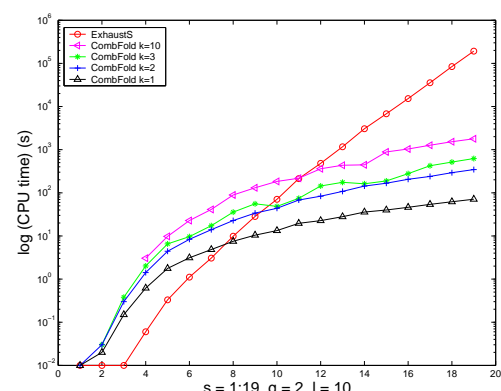

(a)

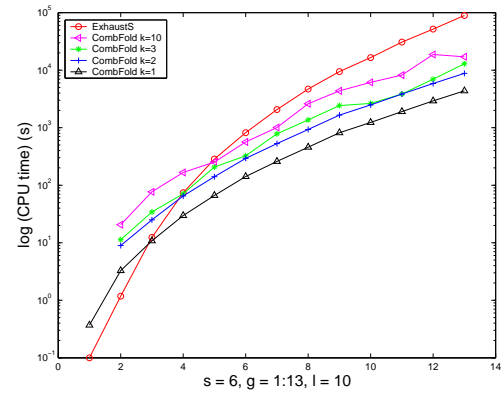

(c)

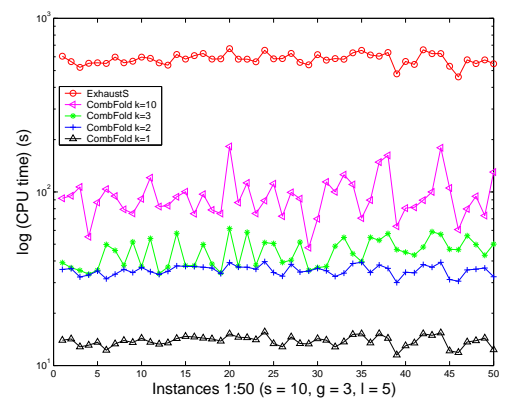

(e)

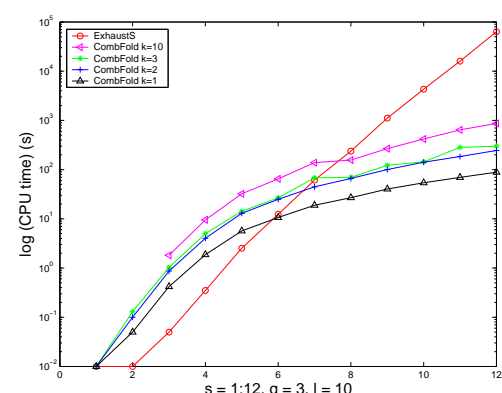

(b)

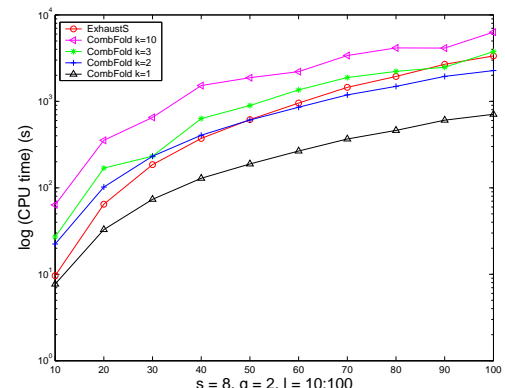

(d)

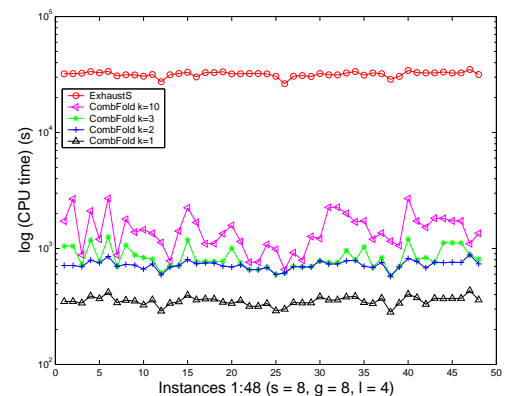

(f)

Fig. 2. Performance of CombFold with $k=1,2,3,10$ and ExhaustS, on sets with different characteristics: (a) 19 instances with $s$ ranging from 1 to 19 , and the same $g=2$ and $l=10$; (b) 12 instances with $s$ ranging from 1 to 12 , and the same $g=3$ and $l=10$; (c) 13 instances with $g$ ranging from 1 to 13 , and the same $s=6$ and $l=10$; (d) 10 instances with $l$ ranging from 10 to 100, and the same $s=8$ and $g=2$; (e) 50 instances with the same characteristics: $s=10, g=3, l=5$; (f) 48 instances with the same characteristics: $s=8, g=8, l=4$. 
$k=1$, and 5-6 times slower than CombFold with $k=10$. For graph (f), where the number of combinations is $8^{8} \approx 16.8 \cdot 10^{6}$ of length 32 , the exponential algorithm is substantially slower, being about two orders of magnitude slower than $\operatorname{CombFold}(k=1)$, and more than one order of magnitude slower than CombFold $(k=10)$.

\section{Conclusions}

We presented here an algorithm that, given a combinatorial set and parameter $k$, predicts the $k$ secondary structures with lowest minimum free energies in the combinatorial set. When the number of words in each set of the overall input-set is considered to be a constant, our algorithm runs in $O\left(s k n^{3}\right)$ time.

In our algorithms, given a combination $C$, we look at the minimum free energy structure only. Extensions of these problems would be to find suboptimal structures (i.e. whose free energy is greater than the MFE), or to consider pseudoknots. Another problem for future work would be to find an algorithm with better running time, for example $O\left(n^{3}+k\right)$.

\section{References}

1. M. Andronescu, Algorithms for predicting the Secondary Structure of pairs and combinatorial sets of nucleic acid strands, M.Sc. Thesis, U. British Columbia, November 2004. http://www.cs.ubc.ca/grads/resources/thesis/Nov03/Mirela_Andronescu.pdf

2. M. Andronescu, D. Dees, L. Slaybaugh, Y. Zhao, B. Cohen, A. Condon, and S. Skiena, Algorithms for testing that sets of DNA words concatenate without secondary structure, Lecture Notes in Computer Science, 2568 Springer 2003, 182-195. Revised version appeared in Natural Computing, 2(4):391-415, 2003.

3. J. V. Ponomarenko, G. V. Orlova, A. S. Frolov, M. S. Gelfand and M. P. Ponomarenko, SELEX_DB: a database on in vitro selected oligomers adapted for recognizing natural sites and for analyzing both SNPs and site-directed mutagenesis data, Nucl. Acids. Res. (2002); 30 (1): 195-199.

4. M. Zuker and P. Stiegler, Optimal computer folding of large RNA sequences using thermodynamics and auxiliary information, Nucl. Acids. Res. (1981) 9: 133-148. 